\title{
Association of Alcaligenes Faecalis Strain in Juvenile Earthworms, from Cocoons of Eudrilus Eugeniae
}

\author{
Ganapathy Nadana Raja Vadivu, S. Sheik Asraf, Karuppaiah Palanichelvam
}

\begin{abstract}
Cocoons of earthworm Eudrilus eugeniae were collected from vermiculture bed and found that it had antibacterial activity. The size of zone of inhibition was directly proportional to the size of cocoons examined. Along with nutritious fluid and embryos, culturable bacterial community was found inside the cocoons. Bacterial colonies were isolated from the trails of newly hatched, juvenile worms in the nutrient agar medium and examined. Gram negative, rod shaped bacterium was found to be abundant in the trails of juvenile earthworms. Polymerase chain reaction was performed from this bacterium to amplify the gene of $16 S$ rRNA and analyzed. Subsequent bi-directional DNA sequencing revealed that this abundant bacterium is highly related to $16 \mathrm{~S}$ rRNA gene sequence of a strain, Alcaligenes faecalis. Based on available literature, we hypothesize that this bacterium could be symbiotically associated with cocoons of earthworms.
\end{abstract}

Keywords: - Earthworm, Eudrilus eugeniae, Cocoon, Alcaligenes faecalis, 16S rRNA sequence.

\section{INTRODUCTION}

Vermicompost and vermiwash, generated by earthworms, were shown to promote the growth of plants. Vermicompost and vermiwash were already shown to have diverse bacterial population and beneficial to agriculture and in waste management [1]. Nutrient-rich vermicompost is generated from organic materials with the combined actions from microorganisms and earthworm [2]. Interestingly, it has been reported recently that there were changes in the composition of bacterial communities and their function during the process of vermicomposting [2]. These changes might be the reasons for the beneficial properties of vermicompost in plant growth. Besides vermicompost, coelomic fluid of earthworms has also been shown to improve the growth of seedlings [3].

Ecosystem of earthworm includes microorganisms, both inside and outside of the body [4]. They belong to a class of invertebrates that produces egg capsules, are also called as cocoons. Cocoons are developed externally over the body of the worms and released through anterior part to the soil. Cocoons are filled with nutrients and embryos; protect themselves in the soil among the microorganism. Cocoons of

\section{Revised Manuscript Received on December 15, 2019.}

Ganapathy Nadana Raja Vadivu, Department of Biotechnology, School of Bio and Chemical engineering, Kalasalingam Academy of Research and Education, Krishnakoil-626126, Email: nadanarajavadivu@klu.ac.in

S. Sheik Asraf, Department of Biotechnology, School of Bio and Chemical engineering, Kalasalingam Academy of Research and Education, Krishnakoil-626126. Email: s.sheikasraf@klu.ac.in

*Karuppaiah Palanichelvam*, Department of Biotechnology, School of Bio and Chemical engineering, Kalasalingam Academy of Research and Education, Krishnakoil-626126. Email: palanichelvam@klu.ac.in
Eisenia fetida, filled with nutritious albuminous fluid, had been shown to possess antibacterial activity [5]. Different levels of lysozyme-like activity were found from homogenized cocoons of earthworm, Dendrobaena veneta [6]. However, it was shown that bacterial population was present inside the cocoons of Eisenia fetida along with developing embryos [7]. Microbiome analysis in the cocoons of Eisenia andrei and Eisenia fetida demonstrated the presence of diverse bacterial population [8]. This association with bacteria has been suggested as beneficial to earthworms in adaptation to the environment. Microbial association of cocoons from earthworm Eudrilus eugeniae has not yet been reported. The cocoons of E. eugeniae have an irregular oval shape, one side usually being flatter than the other.

We report here that cocoons of Eudrilus eugeniae have antibacterial activity. Juvenile earthworms that hatch out from cocoons are surrounded by microbial community. Bacterial species of Alcaligenes appeared to be present predominantly in the body of juvenile earthworms that hatch out from cocoons. The possible interactions between bacteria and earthworms are discussed.

\section{MATERIALS AND METHODS}

A. Earthworm culture and cocoons

Earthworms, Eudrilus eugeniae, were collected from Vermiculture unit from Tirunelveli, Tamil Nadu, India. The collected worms were maintained in the bed prepared with dried leaves, cow dung and soil. The moisture content was continuously monitored by spraying water. Cocoons were collected from the bed and examined. Cocoon was collected on $0.5 \mathrm{~mm}$ mesh sieve. Sizes ranging from 3 to $6 \mathrm{~mm}$ were chosen for study. The cocoons were washed gently in distilled water to remove debris adhering to the sticky hull. Three different sizes of cocoons were kept in LB medium that was plated with Escherichia coli to perform zone of inhibition test.

To initiate hatching of cocoons, wells were created in LB agar medium and sterile distilled water was added to keep them hydrated. Cocoons were observed for every three hours until hatching. Newly hatched juvenile worms were allowed to move over LB agar medium for two hours and removed. Plates were incubated for 12 hours at $37^{\circ} \mathrm{C}$ to identify the bacterium in the trails of worms. To collect the fluid from cocoon, it was sliced in one corner with sterile surgical blade. 
The fluid collected from the cocoon using the micro-pipette tip was mixed with saline solution and plated in LB medium. Bacteria purified from serial dilution plating method were stained with Gram staining kit purchased from HiMedia and followed manufacturer's instructions. Stained cells were visualized using light microscope and documented.

\section{B. PCR and $16 \mathrm{~S}$ rRNA gene sequencing}

Polymerase Chain Reaction was performed using Genomic DNA isolated and subsequent DNA sequencing was performed in Chromous Biotech Pvt. Ltd., Bangalore, India. Two universal 16S primers (Forward: 5'-AGHGTBTGHTCMTGNCTCAS -3') and (Reverse: 5'TRCGGYTMCCTTGTWHCGACTH -3') were used for amplification of $16 \mathrm{~S}$ rRNA gene. The amplified PCR fragment was sequenced bi-directionally, using ABI3500 Genetic Analyzer. The DNA sequence obtained was submitted in NCBI database with accession number MN103745 and named as Alcaligenes faecalis Ec_Af.

\section{Bioinformatics analysis}

Sequenced DNA was blasted with available $16 \mathrm{~S}$ rRNA database using BLASTn program available in National Centre for Biotechnology Information (NCBI). Multiple sequence alignment was performed using Clustal omega program. Phylogenetic tree was constructed by using BLAST pairwise alignments method.

\section{RESULTS AND DISCUSSION}

To understand whether cocoons have any mechanism to protect themselves against other organisms in the soil, antibacterial test against $E$. coli was carried out. Interestingly zone of inhibition was observed near cocoons and the level of clearance was directly proportional to the size of the cocoon as shown (Fig.1). Size of the cocoons is directly connected to the number of juvenile worms that hatch out. It suggested that contents inside the cocoon might be the cause for antibacterial activity. Besides, cocoons are filled with albuminous fluid released from clitellar gland and fertilized eggs [9]. Cocoons were also reported to have culturable bacteria inside [7]. Interestingly, lysozyme-like activity has been reported from homogenized cocoons of Dendrobaena veneta against bacteria [6]. Besides, antibacterial activity has been shown from albumen collected from the cocoons of Eisenia fetida [5]. The zone of inhibition observed from cocoons of $E$. eugeniae (Fig 1) could be due to the lysozyme-like enzyme, albumen or other molecules transuded from cocoons. Matured cocoons were kept in wells made and filled up with sterile distilled water in LB agar plates and were allowed to hatch. Three juvenile earthworms were hatched out and started having mobility in the LB agar medium (Fig. 2). Cocoons appeared to sense the favourable environment to hatch out. The rate of hatching of cocoons was higher in environment having cattle manure compared to sterile distilled water [10]. Cocoons that were smaller in size and not having any juvenile earthworms did not generate any zone of inhibition (data not shown). The nutrients present inside those cocoons might have been depleted by invasion of pathogens or insects. It suggests that molecules like albumen or other compounds present inside the cocoons might play a major role in determining antibacterial activity.

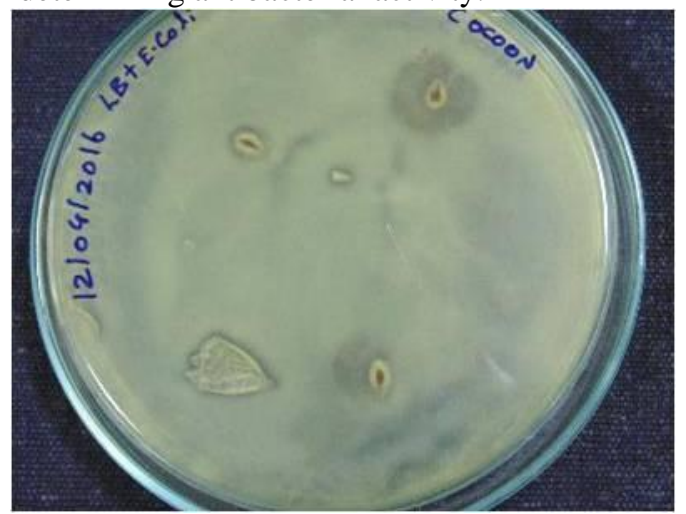

Fig 1. Zone of inhibition by cocoons of E. eugeniae. Photograph was taken after 18 hours incubation of cocoons in LB agar medium plated with $E$. coli strain.

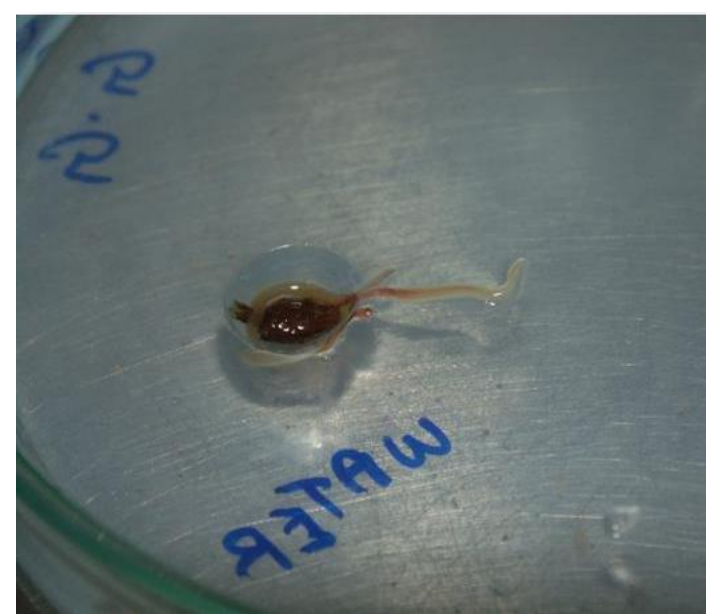

Fig 2. Hatching of cocoons. Photograph was taken after releasing of juvenile worms from cocoons. Three juvenile worms hatched from one cocoon is shown.

To identify the predominant bacterium that is associated with juvenile earthworms, hatched out worms were allowed to move in the LB plate and removed. The plates were incubated for 12 hours and found out bacterial growth in the trail of juvenile earthworms (Fig 3). Moreover, the cocoon fluid that was suspended in sterile saline solution and plated in LB agar medium showed the presence of abundant bacteria (data not shown). Our results clearly indicate that the cocoon fluid has culturable bacterial population as reported earlier [7]. Amplification and sequence analysis of 16S rRNA genes of cocoons of Eisenia andrei and E. fetida revealed the presence of 275 and 176 bacterial species, respectively [8]. Hence, there might be many different bacterial species in the cocoon fluid in E. eugeniae as well and few may be closely associated with juvenile earthworm body. The bacterial population inside the cocoon might be having a mechanism to protect themselves against the antibacterial activity. Earthworms have been reported to have symbiotic association with different microbes. The bacterium, Bacillus endophyticus, present inside the gut region of E. eugeniae has been reported to produce riboflavin, 
which helps in regeneration of organs in worms when amputated [11]. Thakuria et al., 2009 had reported that the association between earthworm and bacteria in gut wall region is based on natural selection process [12].

The selection is determined based on ecological group and availability of food resources in an environment. Many reports are available on symbiotic association of the bacterium, Verminephrobacter in excretory organs (nephridia) of earthworm that belongs to family Lumbricidae. Interestingly it was found that worms with this bacterium reached sexual maturity earlier and had higher rate of cocoon production and its hatching [13]. Candidatus Nephrothrix has been reported to associate with nephridia to play similar role with earthworm Eisenia andrei [14]. Moreover, these bacteria are transmitted vertically to next generation during embryogenesis and cocoon production [15]. Newly developed cocoons of Eisenia fetida were shown to have bacterial consortium and was suggested to influence earthworms' competitive ability [16]. Cocoons of Eisenia species harbored large fraction of symbionts that are transferred from parents compared to horizontal transfer from the environment [8].

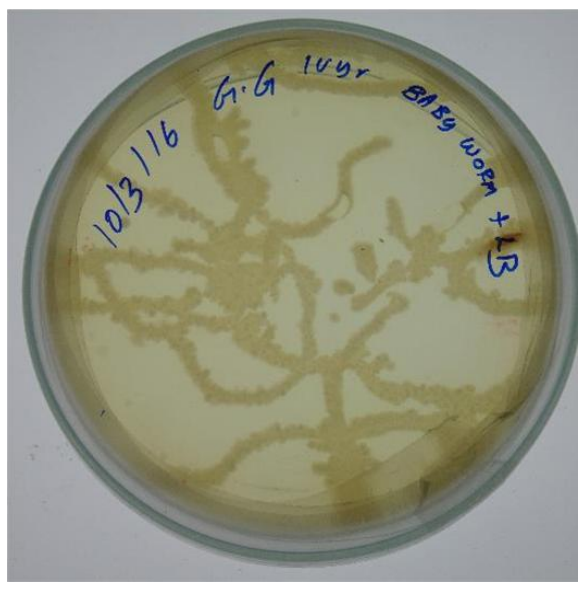

Fig 3. Bacterial growth in juvenile earthworms. Growth of bacteria is shown in the trails of juvenile earthworms after 12 hrs of incubation.

Bacterial association with cocoons or juvenile worms of $E$. eugeniae has not been reported so far. We report here that cocoons of E. eugenieae fluid have culturalble bacteria. Bacterial colonies found in the trails of juvenile worms were serially diluted and plated on LB agar plate. The colony that was morphologically similar and abundant was chosen for Gram staining and found that it was Gram negative rod shaped bacterium (Fig. 4). Zone of inhibition assay was carried out to find out whether this bacterium was producing any antibacterial compound. Significant antibacterial activity was not observed from the bacterial strain isolated (data not shown). Consequently, polymerase chain reaction was performed to amplify $16 \mathrm{~S}$ rRNA gene from a purified colony using primers as described in methods section. Amplified PCR fragment was sequenced and blasted using the program negative bacilli bacterium, Alcaligenes faecalis strain NBRC 13111 rRNA gene sequence. Multiple sequence alignment using Clustal omega program showed that our query sequence has high percentage of identity with species of Alcaligenes BLASTn from NCBI. There was $99.50 \%$ identity with Gram

(Fig 5). Phylogenetic tree was constructed using BLAST pairwise alignments in which our query sequence formed a clade with $16 \mathrm{~S}$ rRNA gene sequence of $A$. faecalis strain NBRC 13111 (Fig 6). We submitted the sequence in NBCI database as described in methods section.

The coccon fluid of E. eugeniae might have diverse strains and Alcaligenes faecalis could be one of them. Hence this bacterium is predominantly present in the bodies of juvenile worms; it might have symbiotic association with the host.

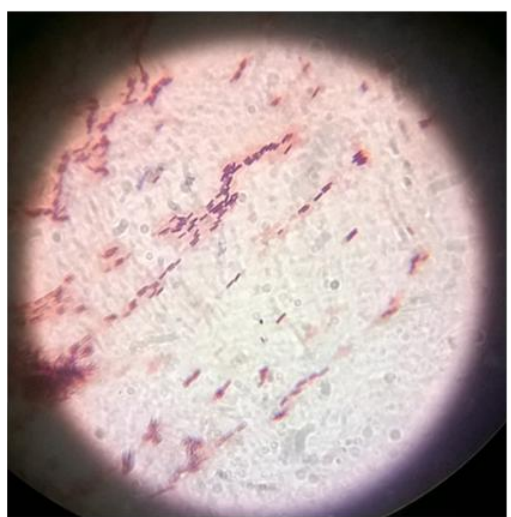

Fig 4. Gram staining of bacteria. Gram negative, rod shaped bacteria was observed in light microscope and photographed.

Although A. faecalis, Gram negative bacterium found initially in feces, it is widely distributed in environment including soil and water [17]. It has been reported as one of the plant growth promoting rhizobacteria. Induction of root growth by a strain of .A faecalis, has been related to its ability to produce plant growth hormone Indole-acetic acid [18]. Moreover, it has been shown to significantly improve the growth of rice plants and induce resistance against fungal pathogens Rhizoctonia solani and Magnaportha oryzae [19]. Species belong to Alcaligenes has been reported from the earthworm castings of Lumbricus terrestris and Aporrectodea caliginosa [20]. It indicates that this bacterium is closely associated with earthworm and beneficial to the plants. However, we report here for the first time that the presence of $A$. faecalis inside the cocoon and predominant in the body of juvenile earthworms of E. eugeniae. Strains of A. faecalis showed various traits such as antimicrobial activity, denitrification and bioremediation of toxic pollutants [21]. It has shown that one of the strains of $A$. faecalis can detoxify the pesticide, endosulfan, in the soil [22]. Interestingly, one of the stains has nematicidal activity by secreting the enzyme serine protease and was suggested to control root knot nematodes in agriculture [23]. Besides, one of the A. faecalis strains has recently been shown to degrade Vitamin B3 very efficiently [21]. Some organisms can use it as carbon or nitrogen source following the degradation. These literatures strongly suggest that earthworm can get benefit from association with this bacterium. This symbiotic association might be determined by the kind of adaptation required in an environment and availability of particular strain. Biochemical characterization and whole genome sequencing of this particular strain might shed more light on basis of this association with earthworm. Besides, more knowledge on this bacterium could lead to 
new biotechnological applications.

\begin{tabular}{|c|}
\hline 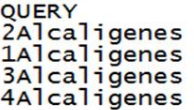 \\
\hline 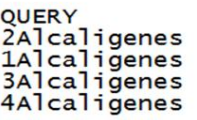 \\
\hline 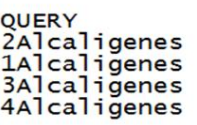 \\
\hline 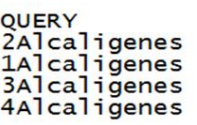 \\
\hline 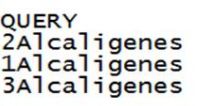 \\
\hline
\end{tabular}

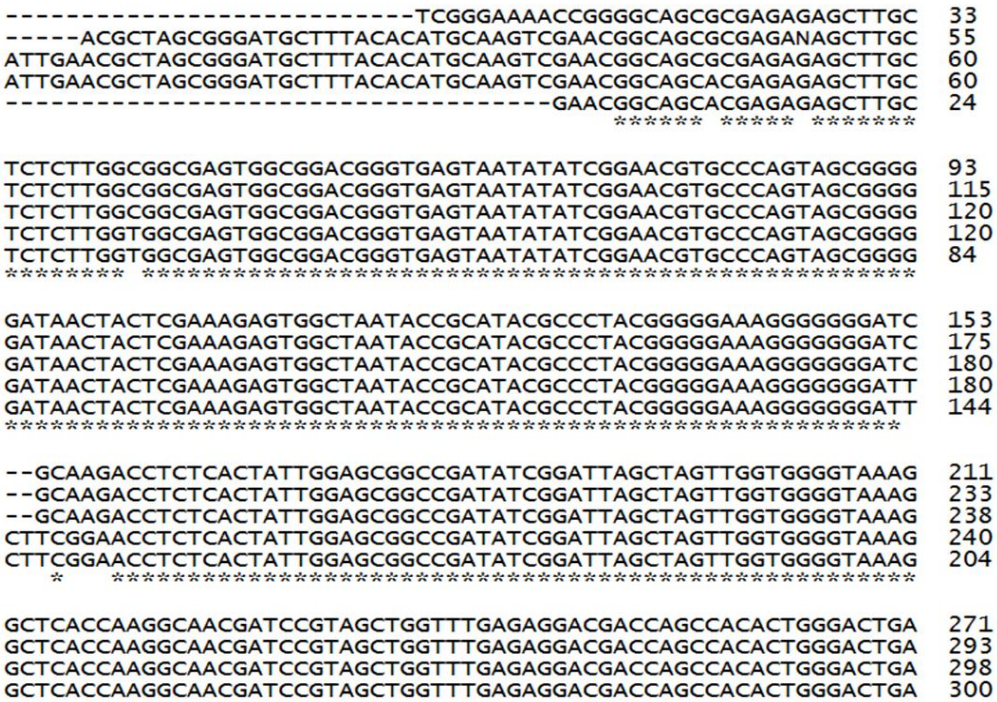

Fig 5. Multiple sequence alignment with Clustal omega program. High percentage of identity was found with four different sequence submissions of Alcaligens faecalis strain

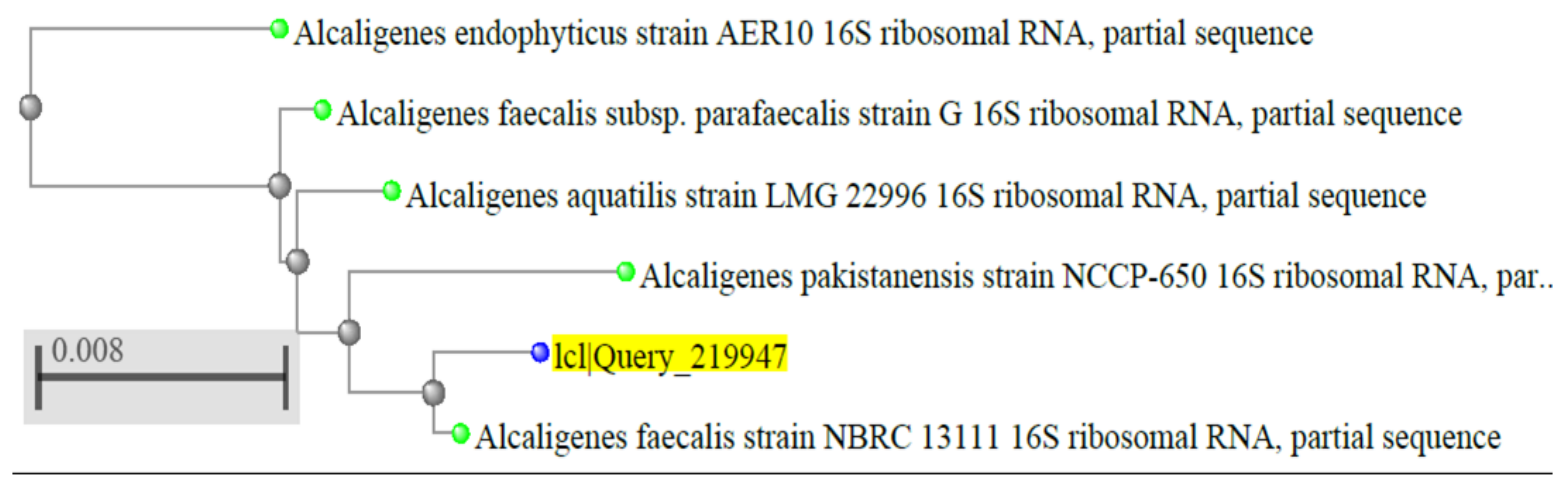

Fig 6. Phylogenetic Tree. BLAST pairwise program from NCBI showing the clade with $A$. faecalis strain NBRC 13111. Bar, represents evolutionary distance scale

bacteria in cocoons of the earthworm Eisenia fetida." Appl. Environ. Microbiol. 59.6 ,1993 , pp. 1904-1910.

\section{ACKNOWLEDGMENT}

This research was carried out with the seed money received from Kalasalingam Academy of Research and Education (KARE), deemed to be university, Krishnankoil, Tamil Nadu, India.

\section{REFERENCES}

1. Pathma, Jayakumar, and Natarajan Sakthivel. "Microbial diversity of vermicompost bacteria that exhibit useful agricultural traits and waste management potential." SpringerPlus 1.1, 2012 , pp. 26.

2. Dominguez, Jorge, M. Aira, and M. Gomez-Brandon "Vermicomposting: earthworms enhance the work of microbes.[in:] Microbes at Work: from Wastes to Resources.[eds:] Insam H., Franke-Whittle I., Goberna M." , 2010 , pp. 93-114.

3. Vadivu, Ganapathy Nadana Raja, Senthamarai Kannan Balaji, and Karuppaiah Palanichelvam. "Putative volatiles in coelomic fluid of earthworm regulate the growth of Vigna radiata." Biocatalysis and agricultural biotechnology 18,2019 , pp. 101070.

4. Lee, Kenneth Ernest. Earthworms: their ecology and relationships with soils and land use. Academic Press Inc., 1985.

5. P. Valembois, P.h. Roch, M. Lassegues, P. Cassand, "Antibacterial activity of the hemolytic system from the earthworm Eisenia fetida andrei." Journal of invertebrate pathology 40.1,1982, pp. 21-27. immunodetection of lysozyme in earthworm Dendrobaena veneta (Annelida)." Journal of invertebrate pathology 109.1 ,2012, pp. 83-90.

7. Zachmann, Joseph E., and J. A. E. Molina. "Presence of culturable
6. M.J. Fiołka, M.P. Zagaja, M. Hułas-Stasiak, J. Wielbo,. "Activity and

8. Aira, Manuel, and Jorge Domínguez. "Optimizing vermicomposting of animal wastes: Effects of rate of manure application on carbon loss and microbial stabilization." Journal of Environmental Management 88.4 ,2008, pp. 1525-1529.

9. Domínguez, Jorge, and Alberto Velando. "Sexual selection in earthworms: Mate choice, sperm competition, differential allocation and partner manipulation." Applied soil ecology 69,2013 , pp. 21-27.

10. Reinecke, A. J., and S. A. Viljoen. "Reproduction of the African earthworm, Eudrilus eugeniae (Oligochaeta)—cocoons." Biology and fertility of soils $7.1,1988$, pp. 23-27.

11. E.R. Subramanian, D. Sudalaimani, J.R. Christyraj, J.D. Christyraj, K. Renganathan, S. Krishnan, S. Sivasubramaniam, "Studies on organogenesis during regeneration in the earthworm, Eudrilus eugeniae, in support of symbiotic association with Bacillus endophyticus." Turkish Journal of Biology 41.1, 2017 , pp. 113-126.

12. D. Thakuria, O. Schmidt, D. Finan, D. Egan, F.M. Doohan, "Gut wall bacteria of earthworms: a natural selection process." The ISME journal 4.3,2010, pp. 357.

13. M.B. Lund, M. Holmstrup, B.A. Lomstein, C. Damgaard, A. Schramm, "Beneficial effect of Verminephrobacter nephridial symbionts on the fitness of the earthworm Aporrectodea tuberculata." Appl. Environ. Microbiol. $76.14,2010$, pp. 4738-4743.

14. F. Viana, L.C. Paz, K. Methling, C.F. Damgaard, M. Lalk, A. Schramm, M.B. Lund, "Distinct effects of the nephridial symbionts Verminephrobacter and Candidatus Nephrothrix on reproduction and maturation of its earthworm host Eisenia andrei." FEMS microbiology ecology 94.2 , 2017, pp. 178. 
15. Davidson, Seana K., and David A. Stahl. "Selective recruitment of bacteria during embryogenesis of an earthworm." The ISME journal 2.5 , 2008 , pp. 510

16. Davidson, Seana K., Ryan J. Powell, and David A. Stahl. "Transmission of a bacterial consortium in Eisenia fetida egg capsules." Environmental microbiology 12.8 ,2010 , pp. 2277-2288.

17. Rehfuss, Marc, and James Urban. "Alcaligenes faecalis subsp. phenolicus subsp. nov. a phenol-degrading, denitrifying bacterium isolated from a graywater bioprocessor." Systematic and applied microbiology 28.5, 2005 , pp. 421-429.

18. D.E.C. Suarez, A. Gigon, R. Puga-Freitas, P. Lavelle, E. Velasquez, M. Blouin,. "Combined effects of earthworms and IAA-producing rhizobacteria on plant growth and development." Applied soil ecology 80 ,2014, pp. 100-107.

19. K.U. Kakar, Z. Nawaz, Z. Cui, A.A. Almoneafy, R. Ullah, Q.Y. Shu. "Rhizosphere-associated Alcaligenes and Bacillus strains that induce resistance against blast and sheath blight diseases, enhance plant growth and improve mineral content in rice." Journal of applied microbiology $124.3,2018$, pp. 779-796.

20. T.Y. Nechitaylo, M.M. Yakimov, M. Godinho, K.N. Timmis, E. Belogolova, B.A. Byzov, , A.V. Kurakov, D.L. Jones, P.N. "Effect of the earthworms Lumbricus terrestris and Aporrectodea caliginosa on bacterial diversity in soil." Microbial ecology 59.3 ,2010, pp. 574-587.

21. Y. Zhang, Q. Chen, J. Ji, L. Zhao, L. Zhang, J. Qiu, J. He. "Complete genome sequence of Alcaligenes faecalis strain JQ135, a Bacterium capable of efficiently degrading nicotinic acid." Current microbiology $75.12,2018$, pp. 1551-1554.

22. L. Kong, S. Zhu, L. Zhu, H. Xie, K. Wei, T. Yan, J. Wang "Colonization of Alcaligenes faecalis strain JBW4 in natural soils and its detoxification of endosulfan." Applied microbiology and biotechnology 98.3, 2014 , pp. 1407-1416.

23. S. Ju, J. Lin, J. Zheng, S. Wang, H. Zhou, M. Sun. "Alcaligenes faecalis ZD02, a novel nematicidal bacterium with an extracellular serine protease virulence factor." Appl. Environ. Microbiol. 82.7 , 2016, pp . 2112-2120. 